Poznańskie Studia Teologiczne 28(2014), s. 115-130.

doi: $10.14746 /$ pst.2014.28.7

Monika Adamczyk

Uniwersytet Pedagogiczny

Kraków

\title{
Zwischen Endlichkeit und Ewigkeit. Adolf Reinachs Konzept des religiösen Erlebnisses
}

Sein ganzes Leben hindurch ist der Mensch ,auf dem Weg“ - auf dem Weg zu sich selbst, auf dem Weg, die Fülle seines eigenen Daseins zu leben. Das menschliche Dasein ist nämlich kein statisches, sondern ereignet sich jeden Tag aufs Neue, aus seinem eigenen Wesen zur Existenz drängend. Für die einen scheint dies erreichbar z. B. durch Prestige oder Karriere, Geld oder Macht, für andere ist es jedoch unbedingt mit dem Absoluten und Notwendigen verbunden. $\mathrm{Zu}$ letzteren zählten jene jungen Menschen, die sich vor über einhundert Jahren in Göttingen um Edmund Husserl versammelt hatten, an seiner Seite die Möglichkeit suchend, das zu erblicken, was wirklich echt und authentisch ist. Sie übten sich in der Kontemplation jedes Gegenstandes und strebten danach, sich nur von der Wahrheit leiten zu lassen. Viele von jenen, die diesen Weg beschritten, haben letztendlich vor dem Gottesantlitz gestanden. Unter ihnen war auch Adolf Reinach.

\section{Der Lehrer des phänomenologischen Ethos}

Adolf Bernhard Philipp Reinach wurde am 23. Dezember 1883 in Mainz als Sohn von Geschäftsleuten geboren. Schon im Gymnasium war er von der Philosophie fasziniert, besonders von Platon, dessen Dialoge er mit großem Entzücken noch auf griechisch gelesen hat. Obwohl er 1901 als Hauptfach seines Studiums Jura wählte, interessierte er sich von Anfang an auch für Vorlesungen in Philosophie und Psychologie. Schon bald wurde er Mitglied des „Akademischen Vereins für Psychologie“, dessen wissenschaftlicher Betreuer Theodor Lipps war. In diesem Verein lernte Reinach Johannes Dauert kennen, der ihn und seine Freunde in der darauffolgenden Zeit an Husserls Logische Untersuchungen heranführte, die Reinach sowie allen seinen Kommilitonen eine neue Welt eröffneten - die Welt des absoluten und echten Seins. Viele dieser jungen Leuten waren so begeistert, dass sie sich für das Studium der Phänomenologie von ihren Quel- 
len an entschieden, in der Annahme, dass sie nur in Göttingen alle notwendigen Werkzeuge erhalten könnten, um einmal die Wahrheit zu erblicken. Unter ihnen war auch Adolf Reinach, der seine Ausbildung von 1905 an unter dem wachsamen Auge von Husserl fortführte ${ }^{1}$.

Mit der Pfiffigkeit und dem Scharfsinn seines Geistes zog Reinach schon bald die Aufmerksamkeit des großen Phänomenologen auf sich. Schon während seines Studiums begründete er mit Theodor Conrad die „Philosophische Gesellschaft Göttingen“, die sich jede Woche im Arbeitszimmer des „Meisters“ traf, um verschiedene philosophische Fragen zu diskutieren. In diesem Studentenverein wuchs die Zukunft der Phänomenologie heran, mit Winthrop Bell, Hedwig Conrad-Martius, Dietrich von Hildebrand, Roman Ingarden, Jean Hering, Fritz Kaufmann, Hans Lipps, Alexandre Koyré und natürlich Edith Stein. Als begabtester Schüler von Husserl wurde Reinach bald auch dessen Mitdenker und Mitforscher und übernahm sogar die Rolle des Lehrers für die neu in Göttingen angekommenen Anhänger der Phänomenologie, sie so als Erster ins Ethos des phänomenologischen Denkens einführend. Für viele von ihnen war er ein Lehrer aus Berufung, in dessen Erscheinung sich nicht nur seine sachlichen und didaktischen Fähigkeiten ausdrückten, die außergewöhnlich waren ${ }^{2}$, sondern auch die Tiefe

\footnotetext{
${ }^{1}$ Die Ankunft der Münchner Phänomenologen in Göttingen nennt man in der Phänomenologiegeschichte die „Münchner Invasion“. Unter diesen Studenten waren außer Adolf Reinach unter anderem Johannes Daubert, Theodor Conrad, Hedwig Martius und Dietrich von Hildebrand (Vgl. K. Schuhmann, Husserl-Chronik. Denk- und Lebensweg Edmund Husserl, w: Husserliana: Edmund Husserl Dokumente, Bd. 1, Haag 1977, s. 89).

${ }^{2}$ Roman Ingarden hat Reinachs Wesen so charakterisiert: „Er war ein guter Lehrer und vor allem glänzender Leiter der philosophischen Übungen. [...] Klar und scharf waren die von ihm gegebenen Problemformulierungen, klar, präzis und kurz gefasst waren die Antworten, die er den Teilnehmern der «Übungen» gab, schlagend waren die Zurückweisungen, mit denen er seinen Standpunkt verteidigte, lebendig und überzeugend die Beispiele, die er anzuführen wusste. Und was besonders kostbar war, war der Umstand, dass er die Fähigkeit hatte, unsere oft ungeschickt formulierten Fragen oder Behauptungen sofort richtig zu verstehen und in den richtigen Problemzusammenhang hineinzustellen. Der Gang der Diskussion war den Teilnehmern überlassen, Reinach selbst fungierte anscheinend bloß als der Hüter, dass man nicht auf Abwege geriet. Im Grunde aber war er das Herz der gemeinsamen Arbeit, der lebendige, gerade in schöpferischer Einstellung neue Forschungswege und Aspekte eröffnende Geist, der seine Aktivität, sein Zugreifen in schwierigen Situationen, seine Geistesgegenwart nie verlor. So war man durch ihn in die Einstellung schöpferischen Philosophierens gebracht, und man konnte sich der Teilnahme am Werden einer neuen Philosophie erfreuen, so sehr man doch in Wirklichkeit ein philosophisches Kind war." (R. Ingarden, Erinnerungen an Husserl, in: E. Husserl, Briefe an Roman Ingarden, hrsg. R. Ingarden, Den Haag 1968, S. 113). Außer mit der Erziehung der nächsten Generation der Phänomenologen, hat er sich intensiv mit der Rechtsphilosophie beschäftigt. Seine Doktorarbeit, die noch in München bei Theodor Lipps geschrieben geworden ist, trägt den Titel Über den Ursachenbegriff im geltenden Strafrecht. 1913 hat er einen Text Über die apriorischen Grundlagen des bürgerlichen Rechts im „Jahrbuch für Philosophie und phänomenologische Forschung“ veröffentlicht, dessen Herausgeber er war. Seine Habilitationsschrift (Wesen und Systematik des Urteils) sowie seine späteren Interessen standen im Bezug zur Sprachphilosophie, besonders beschäftigte ihn hier die Frage des sogen-
} 
seiner Persönlichkeit. Alice von Hildebrand hat in der Biographie ihres Mannes geschrieben: „Vom ersten Augenblick an machte Reinach einen tiefen Eindruck auf Dietrich, der gleich dessen überragende philosophische Begabung und intellektuelle Größe erkannte, die sich mit einer nobeln, attraktiven Persönlichkeit verbanden. Schon nach dieser ersten Begegnung hatte Dietrich große Hoffnungen in ihn gesetzt; doch tatsächlich sollten seine Erwartungen noch weit übertroffen werden. Er spürte Reinachs bedingungslosen Durst nach Wahrheit, seine intellektuelle Disziplin und Gründlichkeit, seine sittliche Prägung. Reinach beeindruckte ihn als eine Person, die vorurteilsfrei offen war für die «Stimme» des Seins. Er besaß eine ungewöhnliche Genauigkeit und Klarheit des Geistes und war in seine Aufrichtigkeit zugleich vertrauenswürdig. Eine Atmosphäre sittlicher Kraft, absoluter Reinheit und ausgesprochen moralischer Größe ging von ihm aus"3. Für viele dieser jungen Leute war die mit Reinach verbrachte Zeit wie bezaubernd, sie inspirierte sie oft zur einsamen intellektuellen Arbeit und zur eigenständigen Suche. Edith Stein, für die Reinach der liebste ihrer Lehrer war, hat geschrieben: „Die Stunden in dem schönen Arbeitszimmer waren die glücklichsten in meiner ganzen Göttinger Zeit. Wir waren uns wohl alle darüber einig, dass wir hier methodisch am meisten lernten. Reinach besprach mit uns die Fragen, die ihn selbst in seiner eigenen Forscherarbeit gerade beschäftigten. [...] Das war kein Dozieren und Lernen, sondern ein gemeinsames Suchen, ähnlich wie in der Philosophischen Gesellschaft, aber anhand eine sicheren Führers. Alle hatten vor unserem jungen Lehrer eine tiefe Ehrfurcht; hier wagte nicht leicht jemand ein vorschnelles Wort, ich hätte kaum gewagt, ungefragt den Mund aufzumachen. Einmal warf Reinach eine Frage auf und wollte wissen, wie ich darüber dachte. Ich hatte angestrengt mitüberlegt und sagte sehr schüchtern in wenigen Worten meine Ansicht. Er sah mich überaus freundlich an und sagte: «So habe ich es mir auch gedacht». Eine höhere Auszeichnung hätte ich mir nicht vorstellen können“4.

Die Aufgabe, die Reinach sich selbst als Lehrer der jungen Phänomenologen stellte, war die Vorbereitung ihres Wesens auf die Erfahrung der absoluten Wahrheit. In diesem Sinne übte er mit ihnen den Gebrauch einer Gedankenaskese, die die unkritische und naive Auffassung des Gegenstands abschüttelt und keine Vorurteile und nichts, was ihre Schau stören könnte, akzeptiert - nicht einmal die vorweggenommene Annahme der Existenz irgendeines zu erforschenden Gegenstands. Zu Beginn des Erkenntnisverfahrens darf man nämlich nicht vorausset-

annten „Sozialen Akts“ (A. Reinach, Über den Ursachenbegriff im geltenden Strafrecht, Leipzig 1905; A. Reinach, Über die apriorischen Grundlagen des bürgerlichen Rechts, München 1953). 3 A. von Hildebrand, Die Seele eines Löwen. Dietrich von Hildebrand, Dusseldorf 2003, S. 47.

${ }^{4}$ E. Stein, Aus dem Leben einer jüdischen Familie, ESGA (= Edith Stein Gesamtausgabe)1, Freiburg 2002, S. 224. 
zen, dass ein Gegenstand existiert oder nicht, weil diese Voraussetzung zwingend das Erkenntnisresultat beeinflussen würde, das sich nicht mit der Seinswahrheit decken muss. Deshalb soll ein Ding als ein mögliches Ding erforscht werden, dessen Existenz oder Nichtexistenz sich erst zeigt, wenn wir seine Wesensanalyse durchführen ${ }^{5}$. Vor diesem Hintergrund hat Reinach immer unterstrichen, dass die Phänomenologie tatsächlich nicht ein System fertiger Wahrheiten oder Theorien darstellt, an die Phänomenologen glauben, dass sie also eben nicht auf Vorurteile gegründet ist, sondern eine Einstellung gegenüber dem Gegenstand. Das Wesen dieser Einstellung ist wiederum das methodische Eindringen des menschlichen Geistes in das innere Sosein des Dings, um dessen Wesen zu ergreifen ${ }^{6}$. Reinach eröffnete seinen Studenten die Sphäre dessen, was a priori ist, indem er ihre konstitutiven Gesetze aufzeigte und beleuchtete. Während eines Vortrags vor Marburger Mathematikern im Jahr 1914 hat Reinach seine Hörer überzeugt: „Was immer an Objekten wir erkennen, sie alle haben ihr «Was», ihr «Wesen», und von allen Wesenheiten gelten Wesengesetze ${ }^{67}$, gleichgültig, ob wir formale oder materielle Gegenstände meinen - Gegenstände aus dem Gebiet der Ethik, Ästhetik, Psychologie, des Rechts oder der Religion ${ }^{8}$. Umso mehr ist alles, was apriorisch ist, zugleich objektiv und notwendig, ,,[...] gleichgültig, welches Bewusstsein sie erfasst. Das Apriori hat an und für sich mit dem Denken und Erkennen auch nicht das mindeste zu tun" "9. Deswegen sind Argumente für die Subjektabhängigkeit der Erkenntnis - dass also alle Erkenntnis vom denkenden Subjekt, das universale und apriorische Schemata auf die Realität projiziert, abhängig ist - falsch. Jede Wahrheit hat apriorischen Charakter nur dank der einprägsamen Notwendigkeit im Sein, die bewirkt, dass ein Gegenstand das ist, was er sein soll. Also nicht Denknotwendigkeit, aber die Wesensnotwendigkeit beherrscht das Reich des a priori. Deswegen: „Wesensgesetze [auf - M. A.] Zusammenhänge beschränken zu wollen durch willkürliche Bindung an die zufälligen Träger, an denen sie sich realisieren, das bedeutet, mit eigener Hand einer Schleier ausbreiten über die Welt der Ideen, in die zu schauen uns vergönnt ist“"10.

${ }^{5}$ Aus diesem Grund war Reinachs Phänomenologie ganz realistisch und die im Geiste von Reinachs Denken erzogenen Phänomenologen waren als „,realistische Phänomenologen“ oder „Reinachs Phänomenologen“ bekannt (K. Schuhmann, Edith Stein und Adolf Reinach, „Studien zur Philosophie von Edith Stein“, 1993 [26/27], S. 63; Husserl und Reinach, w: K. Mulligan, Speech Act und Sachverhalte. Reinach and the Foundations of Realist Phenomenology, Dordrecht 1987, S. 241).

${ }^{6}$ A. Reinach, Sämtliche Werke: Textkritische Aufgabe in 2 Bänden, (Über Phänomenologie), hrsg. K. Schuhmann, B. Smith, Bd. 1, München 1989, S. 541.

${ }^{7}$ Ebd., S. 546.

${ }^{8}$ Ebd, S. 547.

${ }^{9}$ Ebd., S. 545; B. Beckmann-Zöller, Phänomenologie des religiösen Erlebnisses: religionsphilosophische Überlegungen im Anschluss an Adolf Reinach und Edith Stein, Würzburg 2003, S. 76.

${ }^{10}$ A. Reinach, Sämtliche Werke: Textkritische Aufgabe in 2 Bänden, (Rechtsphilosophie), hrsg. K. Schuhmann, B. Smith, Bd. 1, München 1989, op.cit., S. 183; B. Beckmann-Zöller, Phänome- 
Seine Schüler zur redlicher und strenger Erkenntnis erziehend, die keine wissenschaftlichen oder vorwissenschaftlichen Daten über die zu erforschenden Dinge voraussetzt, mit ihnen denkend und philosophierend, hatte Reinach die Bedingungen geschaffen, sein eigenes Wesen für die Absolutheit in ihrer Gänze zu öffnen, nämlich für die Gottesexistenz. Seine Schülerin Hedwig Conrad-Martius, die dieses Ereignis zu explizieren versuchte (sie war selbst Konvertitin) hat gesagt, dass das strengste Argument ungläubiger Menschen die scheinbare Unmöglichkeit der Gottesexistenz sei; der Mensch, der nur mit dem Objekt direkt umgeht, dessen Wesen und mit ihm seine Existenz sich vor ihm selbst zeigt, muss, vor dem Gottesphänomen stehend, sich eigentlich letztendlich der erscheinenden Wahrheit überlassen. Deswegen sollte man sich nicht wundern, dass unerwartet „,...] die große Erkenntnis Gottes über ihn [kam - M. A.]. Es ist verständlich, dass er bis dahin mit unbedingter Ehrfurcht und sachlicher Schau auf Sphären geblickt hatte, die ihre objektive Stellung irgendwo besitzen müssen, die ihm aber persönlich nicht zugänglich gewesen waren. Jetzt aber überströmte ihn dieses Neue und nunmehr in ganz anderem Sinne Absolute mit solcher Fülle und Gewalt, dass sein Blick hier zunächst ausschließlich gebannt wurde" ${ }^{\text {"11 }}$.

\section{Die Rolle des Rätsels im Geschehen der Gottesentdeckung}

Bei Ausbruch des Ersten Weltkriegs meldete sich Reinach trotz seiner zarten Gesundheit als Freiwilliger zum Kriegsdienst. Gerade auf dem Schlachtfeld, unter dem Einfluss der Kriegserlebnisse, hat er Gottesanwesenheit erfahren. Dort nämlich hat sich etwas ereignet, was das Leben dieses Mensch, der in einer völlig areligiösen Familie erzogen worden war, vollkommen veränderte. Über dieses Geschehen schrieb er geheimnisvoll an Husserl: „Wie eine schwere, finstere Nacht liegt die Zeit der großen Offensive hinter mir... Und doch erfüllt mich Glück und unendliche Dankbarkeit, dass ich diese Zeit erleben und überleben durfte. Nun lebe ich in einer ganz anderen Welt ${ }^{“ 12}$. Es ist schwierig, zu sagen, was ihn so sehr zu erschüttern vermochte, aber sicher ist, dass diese Erschütterung eine radikale Wendung nach Innen und zuletzt die Konversion bewirkte ${ }^{13}$. Die Auswirkungen dieser tiefen Kriegserlebnisse haben Reinach höchstwahrscheinlich zu seinen Aufzeichnungen über das religiöse Erlebnis veranlasst. Diese

nologie des religiösen Erlebnisses: religionsphilosophische Überlegungen im Anschluss an Adolf Reinach und Edith Stein, op.cit., S. 74-75.

${ }_{11}$ H. Conrad-Martius, Einleitung, in: A. Reinach, Gesammelte Schriften, Halle 1923, S. XXVII

${ }^{12}$ A. Reinach, Sämtliche Werke: Textkritische Aufgabe in 2 Bänden, (Kommentar zu Aufzeichnungen), hrsg. K. Schuhmann, B. Smith, Bd. 2, München 1989, S. 789.

${ }^{13}$ In Folge dessen wurde Reinach mit seiner Frau während eines Urlaubs protestantisch getauft. 
Notizen bestehen (mit Ausnahme von einigen längeren Textpassagen) aus kurzen, meist wenige Sätze umfassenden Kommentaren, die zwischen dem 24. April und dem 22. Juni 1916 im Schützengraben von ihm verfasst wurden, d. h. bis zu seinem tragischen $\operatorname{Tod}^{14}$.

Reinach war überzeugt, dass der Mensch in schwierigen Situationen am ehesten aufnahmefähig für die Gottesanwesenheit ist, wie z. B. angesichts des nahenden Todes, wenn nichts mehr so klar und selbstverständlich ist wie zuvor und der Mensch in gewisser Weise sich selbst überlassen ist ${ }^{15}$. In der Ruhe und Einsamkeit seines eigenen Inneren, distanziert von Alltagssorgen und Problemen, bemerkt er erst, wie viele grundlegende Fragen ihm bis zu dieser Zeit verborgen waren; wie oft er etwas für seine Existenz Wichtiges und Konstitutives erfuhr, was sich jedoch nicht in Begriffe fassen lässt, die es exakt benennen und explizieren ${ }^{16}$. All das stellt einfach ein Rätsel dar, das der menschlichen Vernunft entgleitet und sich nicht wie ein Puzzlespiel mit Hilfe angemessener Kenntnisse und deren kompetenter Nutzung lösen lässt. Das Rätsel ist unbegreiflich und seine

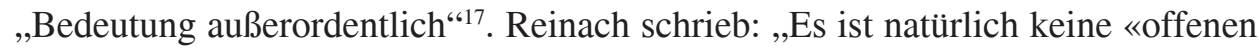

${ }^{14}$ Reinach ist am 1916 während einer Nachtsstreife gestorben. Reinach starb 1916 während einer Nachtsstreife. Vor Reinach hatten über das religiöse Erlebnis auch Wilhelm Windelbrand (Das Heilige, Skizze zur Religionsphilosophie, 1893), Rudolf Otto (Das Heilige, 1917), Wilhelm James (Die Vielfalt religiöser Erfahrung. Eine Studie über die menschliche Natur, 1902) und Karl Girgensohn (Der seelische Aufbau der religiösen Erlebnisses,1921) geschrieben. Nach Reinach schrieb darüber auch ein anderer Phänomenologe, Max Scheler (Vom Ewigen im Menschen), sowie Kurt Stavenhagen (Absolute Stellungnahmen. Eine ontologische Untersuchung über das Wesen der Religion, 1925) und Jean Hering (Phénoménologie et philosophie religieuse. Étude sur la théorie de la connaissance religieuse, 1926). Die letzteren Drei waren höchstwahrscheinlich durch Reinach inspiriert, dessen Notizen im Phänomenologenkreis oft gelesen wurden. Sicher ist, dass Edmund Husserl, Edith Stein, Martin Heidegger, Roman Ingarden und Max Scheler deren Abschrift besaßen. Kurt Stavenhagen sowie Jean Hering wiederum waren Reinachs Schüler in Göttingen (W. Windelbrand, Das Heilige, Skizze zur Religionsphilosophie, Tübingen 1916; R. Otto, Das Heilige, München 1979; W. James, Die Vielfalt religiöser Erfahrung. Eine Studie über die menschliche Natur, Leipzig 1997; K. Girgensohn Der seelische Aufbau der religiösen Erlebnisses, Leipzig 1921; K. Stavenhagen, Absolute Stellungnahmen. Eine ontologische Untersuchung über das Wesen der Religion, Berlin 1925; Jean Hering, Phénoménologie et philosophie religieuse. Étude sur la théorie de la connaissance religieuse, Paris 1926).

${ }^{15}$ Außer durch die Todessituation wird unser Wesen auch durch den Tod anderer und die Notwendigkeit der Übernahme von Verantwortung für jemand anderen für die Gottesexistenz geöffnet. Reinach schrieb: „[...] Du bist verantwortlich und nur Du. Die Menschheit (Volk etc.) ist verantwortlich und Du als ihr Glied. Du bist verantwortlich für Dich und für die Menschheit, der Du angehörst" (A. Reinach, Sämtliche Werke: Textkritische Aufgabe in 2 Bänden, [Aufzeichnungen], op.cit., S. 602). Alle anderen Situationen können unser Wesen nach Reinach sowohl öffnen als auch verschließen.

${ }^{16}$ Vgl. T. Gadacz, Fenomenologia aktu religijnego (Die Phänomenologie des religiöses Erlebnis), in: Demitologizacja, świadectwo, dialog. Niemiecka filozofia religii (Entmythologisierung, Zeugnis, Dialog. Deutsche Religiosphilosophie), red. J. Barcik, G. Chrzanowski, Kraków 2008, S. 166.

${ }^{17}$ A. Reinach, Sämtliche Werke: Textkritische Aufgabe in 2 Bänden, (Aufzeichnungen), op.cit., S. 596. 
Fragen», denn wo vernünftig gefragt werden kann, ist keine absolute Unbegreiflichkeit, sondern allenfalls Unwissenheit. Hier ist aber eine phänomenale Leere evident - eine Ratlosigkeit der Erkenntnis, statt des sich Ausruhens im Erkennen oder des sicheren Strebens im Forschen" ${ }^{18}$. Vielleicht erscheint deswegen alles, was ein Rätsel darstellt, der menschlichen Vernunft als zufällig und irrational, als Antinomie oder als Paradox. Die fehlende Möglichkeit der gedanklichen Erfassung von etwas, die dessen Erkenntnis im Ganzen verhindert, bedeutet aber nicht die Notwendigkeit des Schweigens darüber, weil: ,Jedes Rätsel setzt ein «Datum» voraus. Der Fehler ist, auch dies um der Rätselhaftigkeit willen zu streichen" ${ }^{19}$, besonders, wenn das Rätsel in unserem Leben ein Faktum darstellt und eine geradezu fundamentale Rolle spielt.

Das Rätsel ist nicht transzendent und außerhalb unserer Welt, plötzlich und unerwartet in ihr erscheinend, sondern es ist eher in die Realität eingeschrieben, ist ihr immanent. Mit ihm ist jedes Sein bezeichnet, es pulsiert im Sein und belebt es, geradezu aus ihm sprudelnd. Ein Rätsel stellen wir selbst und ein anderer Mensch dar; rätselvoll ist sowohl, dass wir geboren werden, gebären und sterben, als auch unsere Lebensgeschichte mit unseren Siegen und Niederlagen, unseren Freuden und Nöten - ein Rätsel ist eigentlich die ganze Welt. „Die Welt - schreibt Reinach - wäre ein Rätsel, wenn es keine Rätsel gäbe “20 und „Wenn wir erschauern vor der geahnten Tiefe des Seins und damit vergleichen, was unsere Erkenntnis leistet oder auch nur leisten könnte, so begreifen wir die Rätselhaftigkeit des Seins für uns als notwendig [...] ${ }^{\text {“21 }}$. Unser Dasein unendlich übersteigend, stellt das Rätsel eigentlich nicht etwas Fremdes dar, was uns bedrohen könnte, sondern weist als solches darauf hin, dass jeder von uns ins Zentrum des Geheimnisses der Welt eingesetzt ist, teilnehmend an seinem Mysterium. Dank seiner bemerken wir, dass die Realität nicht so einfach und banal ist, wie sie uns im Allgemeinen scheint, sondern dass ihre Materie ein „Mehr“ in sich birgt. Ist z. B. die Geburt eines neuen Lebens nicht ein Wunder? Oder die Liebe, die unerwartet zu uns kommt? Ist nicht unsere Existenz, allem Unglück zum Trotz, etwas, was uns letztendlich fasziniert und das wir immer intensiver erfahren wollen, um alles, was möglich ist, daraus zu gewinnen? Dennoch kann dieses „Mehr“ nicht aus der vergänglichen Welt stammen, die ständig dem Nichts näherkommt. „Das Irdische ist die Welt des Mehr und Weniger, des Nichts und Einige und Viele, des Werdens und Veränderns und Vergehens“"22, also muss es seine Lebensquelle

\footnotetext{
${ }^{18}$ Ebd., S. 592.

${ }^{19}$ Ebd., S. 602; vgl. T. Gadacz, Filozofia Boga w XX wieku. Od Lavelle'a do Tischnera (Gottes Philosophie in XX Jahrhundert. Seit Lavelle bis Tischner), Kraków 2007, S. 74.

${ }^{20}$ A. Reinach, Sämtliche Werke: Textkritische Aufgabe in 2 Bänden, (Aufzeichnungen), op.cit.,

${ }^{21}$ Ebd., S. 602.

${ }^{22}$ Ebd., S. 608.
} S. 596. 
irgendwo anders haben und sein Übermaß kann nicht „von hier“ kommen. Reinach schrieb: „Die Welt, in der das «mehr oder weniger» waltet, trägt den Stempel des Unzureichenden und Unvollendeten. Das irdisch Unendliche lässt uns mit Evidenz die Unabgeschlossenheit, das nicht in sich Ruhen, das immer Weiterführen erleben. Dagegen aber hebt sich das überirdische Unendliche oder besser das

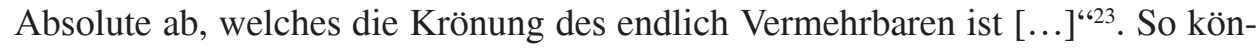
nte man behaupten, dass das Rätsel eine Spur der Ewigkeit darstellt, sehr ähnlich den Chiffren der Transzendenz von Karl Jaspers. Sie geben uns zu denken und weisen nicht nur darauf hin, dass die Ewigkeit tatsächlich existiert, sondern auch darauf, dass sie schon „hier“ zwischen uns geschieht - jedoch nur im Maß ihres endlichen ,Zeichens“" ${ }^{“ 24}$.

Unter den Rätseln, für die Reinach sich persönlich interessierte, war sowohl das schon erwähnte Seinsrätsel als auch das Rätsel der Ahnung, der Antinomie, der Gebetserhörung und der Antinomie der Zeit. Die Ahnung stellt nach Reinach einen intuitiven Einblick des Menschengeistes in verschiedene künftige Inhalte dar. Als solche ist die ,[...] Ahnung - etwa von einem künftigen Ereignis - kein Gefühl [...] wie Freude oder Trauer, keine Ich-Zuständlichkeit, kein sich so oder anders Befinden des Ich. Vielmehr fügt die Ahnung dem Gesamtwissensschatze - im weitesten Sinne des Wissens gesprochen - etwas Neues hinzu; das Subjekt scheint hier, mit Recht oder Unrecht sei dahingestellt, vermittelst der Ahnung etwas aus dem Flusse künftiger Begebenheiten zu erfassen, was ihm vorher nicht

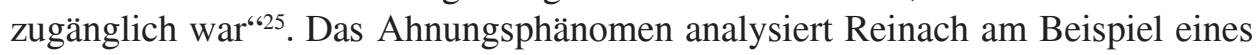
Gespräches seiner Kriegskameraden, die sich an einen gerade gefallenen Freund erinnern. Alle Soldaten waren überzeugt, dass dieser Mensch sich auf den Tod vorbereitet hatte, sein baldiges Eintreten vorausfühlend, obwohl eigentlich nichts darauf hindeutete, denn er erfreute sich einer guten Gesundheit und die Gefahr von Seiten des Feindes war nicht so groß, um deshalb in besonderer Sorge zu sein. Dennoch hatte dieser Soldat am Tag zuvor alle seinen persönlichen Sachen geordnet und sie danach seinen Kameraden übergeben. Mehr noch, er hatte auch einen Abschiedsbrief an seine Frau und seine Kinder geschrieben sowie sein Testament. Man weiß nicht, „wie“ er von seinem baldigen Tod wissen konnte, aber: „Durch die Ahnung erfassen wir - oder glauben wir zu erfassen - vorher Verborgenes. Und es kann auch im Ahnen eine Überzeugung gründen, die an Stärke und innerer Sicherheit der sich auf ein Erkennen stützenden Überzeugung nicht nachzugeben braucht. Aus der Ahnung des nahen Todes erwächst die sichere Überzeugung, bald sterben zu müssen“"26.

${ }^{23}$ Ebd.

${ }^{24}$ Vgl. T. Gadacz, Fenomenologia aktu religijnego, (Die Phänomenologie des religiöses Erlebnis), dz. cyt., S. 166.

${ }^{25}$ A. Reinach, Sämtliche Werke: Textkritische Aufgabe in 2 Bänden, (Aufzeichnungen), op.cit., S. 590-591.

${ }^{26}$ Ebd., S. 591. 
Über die Antinomie der Gebetserhörung dachte Reinach am Beispiel eines Betenden nach, der darum bittet, von einer Lawine verschont zu werden. Wenn die Lawine wirklich nicht niederrollt, erlebt der Betende dies als Folge seines Gebets. Aber vielleicht war es auch so - überlegt Reinach -, dass seine Errettung lediglich die Auswirkung auf der Bahn der Lawine liegender Steine war, dank derer sie ihre Richtung geändert hat? Dann wäre das Erlebnis der Lebensbedrohung nur eine Täuschung gewesen und Gott hätte gar kein Gebet erhört. Aber vielleicht verhielt es sich auch so, dass Gott alles vorausgeschaut hat und das Gebet vorausgehört? Nein, schrieb Reinach, man soll ,[...] den religiösen Erlebnissen ihren Sinn lassen! Auch wenn er zu Rätseln führt. Gerade diese Rätsel sind vielleicht für die Erkenntnis von dem höchsten Werte. Gottes Barmherzigkeit hat mir das Leben geschenkt, das ist der tiefe Hintergrund: und in der Welt spielt es sich ab, indem das Sandkorn die Lawine wegdrängt. Durch das Gebet aber bin ich im Zusammenhang mit jenem letzten Hintergrund der Welt. Freilich, das Gebet ist in der Zeit, und die Erhörung muss ihm folgen - und es ist dieselbe Zeit wie die, in der die Lawine niederfällt. Und doch $[\ldots]^{\text {“27 }}$.

Die Antinomie der Zeit betrifft das Wesen der Gottesexistenz, das auf der einen Seite im Zeitlichen immerwährend ist, so wie in jedem Moment die Wahrheit gültig ist. Auf der anderen Seite kann unsere Existenz jedoch nur zwischen Vergangenheit und Zukunft ausgebreitet sein. Wenn wir die Zeitkategorie auf Gott beziehen, geraten wir deswegen in ein Paradox. Gott ist die ,absolute Person, die von uns weiß, sich unserer erbarmt, uns vergibt, und deren Akte doch nicht als in der Zeit verlaufend, als vergangen oder zukünftig gedacht werden sollen. Gott «hat vergeben» oder «wird» vergeben, hätte danach keinen wörtlich zu nehmenden Sinn“"28, für ihn muss einfach alles in der absoluten Gegenwart geschehen, die jedoch für uns unbegreiflich bleibt.

Das Rätsel verhüllt und versteckt subtil die Wirklichkeitstiefe, es weckt nur manchmal unsere Aufmerksamkeit. Dann erweckt es uns langsam aus Alltagsruhe und Lethargie, und weist uns auf das „Mehr“ hin, das sich ständig in der Welt vollzieht. Obwohl die Rätsel also unsere Aufmerksamkeit fesseln, verweisen sie immer über sich hinaus und schicken uns irgendwo „weiter“. Die Ewigkeit erscheint nur in ihnen, von sich zeugend, die Rätsel sind gleichsam ihre Ankündigung. In diesem Sinn stellt das Rätsel ein Tor dar, das der Mensch durchschreiten muss, um sich für die Gotteserfahrung zu öffnen - eine Schwelle, hinter der sich das religiöses Erlebnis ereignen kann.

\footnotetext{
${ }^{27}$ Ebd., S. 593.

${ }^{28}$ Ebd., S. 601.
} 


\section{Die innerliche Struktur des religiösen Erlebnisses}

Obwohl das Rätsel der Vernunft immer verborgen bleibt und sich nie völlig begreifen lässt, ist es nach Reinach greibar für das Fühlen, das gleichsam ein innerer „Sinn“ unseres Wesens ist. Als solcher stellt es ein intuitives Schauen in die Sphäre der apriorischen und notwendigen Gesetze, Normen, Werte und all dessen, was eigentlich mit der Ewigkeit verbunden ist, dar. Jedoch ist das Fühlen nach Reinach nicht nur ein besonderes Werkzeug der Vernunft, sondern auch ein Medium zwischen dem Menschen und Gott, der sich nur im spezifischen Erlebnis erfahren und erfassen lässt, wie dem Abhängigkeitsgefühl, dem Geborgenheitsgefühl, der Dankbarkeit, dem Vertrauen und natürlich der Liebe ${ }^{29}$. Was ist also das Fühlen eigentlich und wie genau sieht, auf ihm fundiert, das religiöses Erlebnis aus?

Jahrhundertelang wurde die Rolle des Fühlens für die Erkenntnis bestritten, wurde das Fühlen mit den emotionellen Gefühlen verwechselt. Zur Rehabilitation des Fühlens kam es erst im 20. Jahrhundert ${ }^{30}$. Der Unterschied zwischen dem Fühlen und dem Gefühl besteht darin, dass das Gefühl eine passive Empfindung darstellt, während das Fühlen sich immer aktiv auf einen Gegenstand richtet, das Fühlen ist also intentional. Während die Gefühle rein immanent sind und ,in sich selbst verschlossen“, ist das Fühlen transzendent, entdeckt etwas Neues; während die Ersteren im Grunde unsere Aufmerksamkeit auf uns selbst konzentrieren, verweist das Zweite über sich und uns hinaus auf etwas anderes. Das ist auch der Grund, weshalb jemand, der sehr traurig ist oder in einer tiefen Depression gefangen, auf die Frage nach der Ursache seiner schlechten psychischen Kondition wahrscheinlich nicht direkt antworten kann. Es ist so, dass Gefühle ,[...] zwar wie alles Erleben ihre reale Ursache haben, aber keineswegs sich erlebnismässig auf etwas beziehen, dem sie gelten“"31. Die Gefühle umfangen unser Wesen, wir „versinken“ in ihnen; sie überfluten uns fortwährend mit Stimmungen. „Von eigentlicher Erkenntnis ist nur dort zu reden, wo ein Einverfassen von Sachver-

\footnotetext{
${ }^{29}$ In seinen Notizen sagt Reinach nicht so viel über den Unterschied zwischen dem religiösen Erlebnis und dem Glaubensakt, man kann jedoch feststellen, dass, während das Erste zwischen Mensch und Gott eine Beziehung knüpft, im Glaubensakt die Gottesexistenz vom Menschen anerkannt wird. So ist das religiöse Erlebnis fundamentaler, weil der Mensch, wenn er sich an Gott richtet, dies nicht tut, indem er dessen Existenz schon voraussetzt, sondern er entdeckt seine Existenz in dem Moment, in dem er seine Anwesenheit erfährt (Vgl. B. Beckmann-Zöller, Phänomenologie des religiösen Erlebnis: religionsphilosophische Überlegungen im Anschluss an Adolf Reinach und Edith Stein, op.cit., S. 125-128).

${ }^{30}$ Die Rolle des Fühlens für die Erkenntnis hat eigentlich Max Scheler in seinem Werk „Der Formalismus in der Ethik und die materiale Wertethik" wieder begründet (M. Scheler, Der Formalismus in der Ethik und die materiale Wertethik, Halle 1921).

${ }^{31}$ A. Reinach, Sämtliche Werke: Textkritische Aufgabe in 2 Bänden, (Aufzeichnungen), op.cit., S. 603; vgl. Por. M. Scheler, Der Formalismus in der Ethik und die materiale Wertethik, op.cit., S. 264-265.
} 
halten stattfindet, wo etwas Bestehendes «ergriffen» wird, wo ein «Entdecken» möglich ist und ein Wissen, welches im Entdecken und Erfassen seiner Fundierung findet ${ }^{“ 32}$. Dabei darf man hier nicht nur ,[...] an die Fälle, in denen uns ein mathematischer Sachverhalt aufleuchtet oder ein Geschehen in der Sinnenwelt uns anschaulich entgegentritt [reden - M. A.], auch da, wo von uns etwa ethische Werte oder Unwerte lebendig erfasst werden, wo wir den Wert der Liebe oder den Unwerte des Neides aufs innigste erfühlen, auch hier gibt es «Endeckung», «Erfassung» und ein Wissen, das sich darauf gründet" ${ }^{\text {“33. }}$.

Wie schon erwähnt, behauptet Reinach, dass der Mensch sich in Grenzsituationen am ehesten für die Gotteserfahrung öffnet, dann, wenn sein „Sein“ oder „Nichtsein“ in Frage steht. In diesem Moment geschieht, was bis dahin nur möglich schien, mit durchschlagender Evidenz. Der Mensch, nun nicht mehr wie bis dahin außerhalb des Geschehens, sondern in dessen Zentrum, bemerkt erst dann die Schwäche und Vergänglichkeit seines eigenen Daseins, die Unvollkommenheit und Flüchtigkeit seines Wesens. Er fühlt sich, als hätte sich vor ihm ein Abgrund aufgetan und das Leben hätte ihn immer tiefer in dessen Schlund gezogen $^{34}$. Jedoch ist das Grunderlebnis, das den Menschen hier begleitet, nach Reinach nicht eigentlich das der Angst und Sorge um sich selbst, das seine Quelle im Gefühl der physischen Nichtigkeit hat, sondern das Erlebnis der Endlichkeit der eigenen Person. Reinach schrieb: „Das Bewusstsein der Schwäche ist in der Stunde der Todesgefahr erwachsen. [...] Bewusstsein, nichts gegen die Gefahren und drohenden Leiden und den Tod tun zu können. Natürlich kann einem auch dabei das «nicht viel vermögen» klar werden. Aber darum handelt es sich nicht. Man könnte sagen: Erlebnis der absoluten Ohnmacht. Aber ob es das trifft? Es ist doch Erlebnis einer absoluten Kleinheit, rein an sich, ohne Bezug darauf, ob man dieses oder jenes doch ausrichten könnte?"35. Diese Kleinheit der Person, von der Reinach spricht, kann man aber eigentlich nicht in Bezug auf einen materiellen Gegenstand oder ein konkretes Ereignis erleben, sondern eher im Bezug zu einer anderen Person, zum Anderen, der in uns Angst erweckt, weil er stärker und vielleicht sogar mächtiger ist als wir selbst. Und das hat seine Richtigkeit, denn in diesem Moment erfährt der Mensch die Anwesenheit eines Weiteren, der „,in der Nähe“ von ihm steht. Dieser Anderer kommt jedoch nicht in schlechter

\footnotetext{
S. 603 .

${ }^{32}$ A. Reinach, Sämtliche Werke: Textkritische Aufgabe in 2 Bänden, (Aufzeichnungen), op.cit.,

${ }^{33}$ Ebd; vgl. M. Scheler, Der Formalismus in der Ethik und die materiale Wertethik, op.cit., S. 263-263.

${ }^{34}$ Vgl. B. Beckmann-Zöller, Fenomenologia przeżycia religijnego wedtug Adolfa Reinacha i Edyty Stein (Die Phänomenologie des religiöses Erlebnis bei Adolf Reinach und Edith Stein), w: A. Grzegorczyk (red.), Niewidzialna rzeczywistość. Szkice o filozofii duchowej Edyty Stein (Die unsichtbar Realität. Skizze von Geistphilosophie Edith Steins), Poznań 1999, S. 193.

${ }^{35}$ A. Reinach, Sämtliche Werke: Textkritische Aufgabe in 2 Bänden, (Aufzeichnungen), op.cit., S. 605.
} 
Absicht, sondern streckt dem Menschen seine Hand entgegen, um ihn vor dem Abgrund, an dem er steht, zu retten ${ }^{36}$. Dieser jemand - sagt Reinach - ist Gott selbst.

Im Angesicht der Gottesanwesenheit erlebt der Mensch zunächst die eigene Abhängigkeit von Ihm, und zwar dadurch, dass die Gottesmajestät ihn unendlich übersteigert. Wir lesen bei Reinach: „Die Abhängigkeit steckt z. B. immer darin, wenn auch unentfaltet, schon im wesentlichen «absoluten Blick nach oben» "37. Das menschliche Kleinheitserlebnis vertieft in dieser Situation noch mehr die Notwendigkeit des Vertrauens zu Gott, der als Einziger dem Menschen helfen kann. Der Mensch fühlt dann, wie das Erlebnis der Schwäche seines eigenen Wesens gegenüber Gott ihn immer mehr umfängt und fast lähmt. Er träumt davon, allein handeln zu können, nicht auf Gott angewiesen, der ihm fremd und als ein vollkommen anderer erscheint. Wenn er jedoch bereits nicht mehr weiß, was mit ihm geschieht, sich dem vor ihm auftuenden Abgrund nähernd, als ob er bereits langsam fiele, fühlt er gleichzeitig, dass er , in der Hand Gottes steht “38, die ihn birgt und schützt und ihn nicht fallen lässt ${ }^{39}$. Deswegen erscheint die erlebte Abhängigkeit von Gott letztendlich nicht als eine ,,[... Grade die kürzeste Verbindungslinie “40, weil Gott nicht als ein Herrscher zu ihm kommt, sondern als der, der immerfort an ihn denkt und sich um ihn sorgt, immer bereit, ihm sein eigenes Sein zu entdecken. Er ist jemand, der mit dem Menschen in eine Beziehung treten will und nur darauf wartet, wann dieser selbst sich dafür entscheidet. Deswegen passt Er sich an den Menschen an, in gewisser Weise sich selbst hingebend. Deshalb ist das Abhängigkeitgefühl eher der Ausdruck einer Möglichkeit des menschlichen Wesens, nämlich der Möglichkeit des Seins in Gottes Händen, die sich zu einem grundsätzlichen Zustand entwickeln kann, weil die echte Berufung des Menschen ein Leben „,in“ und ,aus“ Gott ist.

Neben dem Abhängigkeitsgefühl entsteht im Menscheninneren auch das Geborgenheitsgefühl, das Quelle der menschlichen Überzeugung ist, dass er nicht nur ,in der Hand Gottes steht“, sondern dass er sich in dessen Händen auch wie ein Kind im Mutterschoss befindet. Gottes Hände stellen für ihn eigentlich zu

\footnotetext{
${ }^{36}$ Vgl. A.U. Müller, Grundzüge der Religionsphilosophie Edith Steins, Freiburg 1993, S. 151.

${ }^{37}$ A. Reinach, Sämtliche Werke: Textkritische Aufgabe in 2 Banden (Aufzeichnungen), op.cit., S. 599; T. Gadacz, Filozofia Boga w XX wieku. Od Lavelle'a do Tischnera (Gottes Philosophie in XX Jahrhundert. Seit Lavelle bis Tischner), op.cit., S. 77.

${ }^{38}$ A. Reinach, Sämtliche Werke: Textkritische Aufgabe in 2 Banden (Aufzeichnungen), op.cit., S. 600 .

${ }^{39}$ B. Beckmann-Zöller, Fenomenologia przeżycia religijnego wedtug Adolfa Reinacha i Edyty Stein (Die Phänomenologie des religiöses Erlebnis bei Adolf Reinach und Edith Stein), w: Niewidzialna rzeczywistość. Szkice o filozofii duchowej Edyty Stein (Die unsichtbar Realität. Skizze von Geistphilosophie Edith Steins), op.cit., S. 192-193.

${ }^{40}$ A. Reinach, Sämtliche Werke: Textkritische Aufgabe in 2 Banden (Aufzeichnungen), op.cit., S. 594.
} 
jeder Zeit und in jeder Lage eine Zuflucht dar, ihn durch das ganze Leben tragend. In ihnen erreicht er die Ruhe und die Fülle seiner Existenz, er will „,nicht weiter führen“"41 und sucht nichts mehr. Sein Wesen ist dann erfüllt mit der Dankbarkeit für die ihm geschenkte Sicherheit und die Möglichkeit der Erreichung der Seinsfülle und das Vertrauen, weil er in diesem Moment sich Gott absolut hingibt, in dem Glauben, dass die Kraft, die ihn birgt, gütig ist ${ }^{42}$. So ist es „Bemerkenswert, wie Abhängigkeits-, Geborgenheits-, Dankbarkeits- etc. -erlebnis miteinander verknüpft sind. Eines scheint das andere $\mathrm{zu}$ fordern, mitunter drinzustecken [...]. Hier scheint mehr eine logische Verknüpfung zu sein, und zwar herrscht hier die Logik des spezifisch religiösen Erlebnisses. [...] Nun haben wir das Erlebnis der Geborgenheit schlechthin, aus dem sich Vertrauen (als bezüglich auf Konkretes) «logisch» entwickelt. Und ebenso die Dankbarkeit aus dem noch tiefer zugrundeliegende Abhängigkeitsgefühl (in der Hand Gottes stehen). Also scheint diese Stufenfolge: Abhängigkeitsgefühl schlechthin - Geborgenheitsgefühl schlechthin. Aus dem ersten entspringt Dankbarkeit, aus dem zweiten konkretes Vertrauen. Das zweite setzt das erste voraus “433. Alle diese Erlebnisse bilden die noematische Dimension des religiösen Erlebnisses, das die Krönung unserer Vereinigung mit Gott darstellt, wenn der Mensch durch Gott zu einem echten und authentischen Leben hingetragen wird. Diese ,eigentliche Vollkommenheit“"44, die der Mensch hier erreicht, ist jedoch nicht identisch mit der Vollkommenheit der ersten Menschen oder einer Notwendigkeit, die in unser Dasein eingeschrieben wäre. Reinach schrieb: „Das «eigentlich vollkommen» muss besonders beachtet werden. Nicht $=$ anfänglich, nicht $=$ seinsollend. Gerade das letztere naheliegende Missverständnis muss vermieden werden. Vielleicht handelt es sich um eine sonst nicht bekannte eigene Kategorie. Vielleicht kann man die Sache vergleichen mit dem Wahnsinnigen, der «eigentlich» viel mehr als: früher gewesen. Die Frau des Wahnsinnigen wird ihn noch immer lieben, ihn, der «eigentlich» ja ganz anders ist, als er eben erscheint ${ }^{\star 45}$. Im religiösen Erlebnis vollzieht sich nämlich eine Transformation des menschlichen Ichs, dank der er zu seinem eigentlichen Sein, das in sein Wesen eingeschrieben ist, zurückfindet ${ }^{46}$.

${ }^{41}$ Vgl. A.U. Müller, Grundzüge der Religionsphilosophie Edith Steins, op.cit., S. 147; B. Beckmann-Zöller, Phänomenologie des religiösen Erlebnis: religionsphilosophische Überlegungen in Anschluss an Adolf Reinach und Edith Stein, op.cit., S. 138.

${ }^{42}$ Vgl. B. Beckmann-Zöller, Phänomenologie des religiösen Erlebnis: religionsphilosophische Überlegungen in Anschluss an Adolf Reinach und Edith Stein, op.cit., S. 138.

${ }^{43}$ A. Reinach, Sämtliche Werke: Textkritische Aufgabe in 2 Bänden (Aufzeichnungen), op.cit., S. 599-600.

${ }^{44}$ Ebd., S. 594.

${ }^{45} \mathrm{Ebd}$.

${ }^{46}$ Vgl. Vgl. B. Beckmann-Zöller, Phänomenologie des religiösen Erlebnis: religionsphilosophische Überlegungen in Anschluss an Adolf Reinach und Edith Stein, op.cit., S. 132. 
Alle Erlebnisse, die sich aus dem religösen Erlebnis begründen, haben absoluten Charakter, im Gegensatz zu den Erlebnissen, die in menschlichen Beziehungen gründen. Während die letzteren zufällig sind oder mit einer konkreten Gesinnungsart verknüpft, also relativ, sind alle Beziehungen zu Gott absolut, weil ihre Intentionsrichtung seinen absoluten Gegenstand absteckt. „Gott thront im Himmel“"47, daher verlaufen sie von „oben“ nach „unten“ und übersteigen die Grenze dieser Welt. In der Transzendenz des religiösen Erlebnisses erhebt Gott den Menschen nach oben, wodurch seine irdischen Beziehungen einen überirdischen Charakter bekommen. Reinach schrieb: „Die Stellung, die wir ihm gegenüber einnehmen, ein absolutes Unten einem absoluten Oben gegenüber, schreibt uns unser erlebnismäßiges Verhalten vor: Vertrauen, Liebe, Abhängigkeit [... $]^{648}$. Diese Absolutheit des religiösen Erlebnisses stellt seine noematische Dimension dar, die „die Brücke zu dem Reiche des Absoluten“49 ist, als solche bestehend aus einer formalen und einer materialen Seite. Reinach behauptet: „In dreierlei natürlich eng Zusammenhängendem Sinne können wir nunmehr von Absolutheit reden. Gott ist uns in absoluter Höhe gegeben; unser einzelnes Erleben trägt demgemäß die absolute Höherichtung; und neben dieser formalen Absolutheit steht die Absolutheit des materialen Gehalts, die gänzliche Erfülltheit des Vertrauens, des Denkens, der Liebe“"50. Zwischen beiden Dimensionen vollzieht sich der Logos des religiösen Erlebnisses, dessen Beziehung nicht auf einer logischen Schlussfolgerung gründet, sondern auf inneren und harmonischen Übergängen sodass: „Dem zuhöchst Gegebenen, zu dem unser Erleben sich emporhebt, ziemt eine absolute Fülle der Liebe; wie auch umgekehrt ein absolutes Vertrauen, das mich erfüllt, seinen intentionalen Gegenstand in absoluter Höhe aufsuchen muß ${ }^{* 51}$.

Ein religiöses Erlebnis kann man jenes Erlebnis nennen, in dem der Mensch die Tatsache seines eigenen Seins in Gottes Händen entdeckt. Diese Entdeckung vollzieht sich nicht auf der Ebene der theoretischen Reflexion, sondern auf der Ebene des Fühlens, die eine innere Dynamik des religiösen Erlebnisses darstellt, dank der es sich verwirklicht. Erkennend, dass Gott mit seinen mächtigen Händen sein vergängliches Sein schützt und ihn stützt, entdeckt der Mensch einen Zustand, in dem er sich sicher fühlt und wo sich seine Wesensfülle entfaltet. Jetzt, da er nicht mehr weiterzugehen oder etwas zu erreichen begehrt, bleibt er in innerer Beziehung zu Gott. Jedoch muss man sagen, dass der Glaube nicht einem ruhigen, sondern einem reißenden Bach gleicht und man kann vermuten, dass

${ }^{47}$ A. Reinach, Sämtliche Werke: Textkritische Aufgabe in 2 Bänden (Aufzeichnungen), op.cit., S. 607.

${ }^{48}$ Ebd.

${ }^{49}$ Ebd., S. 610.

${ }^{50}$ Ebd., S. 607.

${ }^{51}$ Ebd. 
vielleicht schon bald die Notwendigkeit seiner Bestätigung eintritt. Im religiösen Erlebnis aber erkennt der Mensch die Möglichkeit der Kommunion zwischen sich und Gott, er erfährt die Umarmung durch die Liebe des Vaters.

$* * *$

Der Mensch versucht so, seinen Lebenslauf „zu schreiben“, um Herr und Schmied seines Lebens zu sein. Zu vergänglich und schwach, entführt ihn aber mitunter der Lebensstrom und lässt ihn auf eine Sandbank auflaufen. Jedoch öffnet sich der Mensch gerade dort für die Gottesanwesenheit, was Adolf Reinach sehr gut wusste, dem gerade auf dem Schlachtfeld Gott begegnet war. Edmund Husserl sagte in seinem Nachruf, den er am Grab seines großen Schülers und Freundes gehalten hat: ,[...] zu tief war seine religiöse Grundstimmung durch die ungeheuren Kriegserlebnisse betroffen, als daß er in Zeiten eines relativ ruhigeren Frontdienstes nicht hätte den Versuch wagen müssen, seine Weltanschauung religionsphilosophisch auszubauen. Wie ich höre, rang er sich in der Tat zu einer ihn befriedigenden Klarheit durch: Die feindliche Kugel traf den in sich Beruhigten, mit sich und Gott völlig Einigen“"52. Natürlich ist Reinachs Weg zu Gott nicht der einzige und wie er selbst in seinen Notizen schrieb „Jeder kann natürlich nur von dem reden, was er erlebt" ${ }^{\prime 53}$. Und doch muss jeder Weg zu Gott durch das Erlebnis seiner Anwesenheit und Vertrautheit führen, wo der Mensch fühlt, wie nahe ihm Gott ist, der auf seine Entscheidung wartet, und wie viel er eigentlich für Ihn bedeutet. Denn ,[...] nur im Erleben Christi kann die unendliche Ferne und Majestät der Gottheit [...] in die unendliche Nähe verwandelt werden, durch die der Christ im Gebet sich persönlich gehört und aufgehoben weiß"54.

\section{Between Finiteness and Infinity. Adolf Reinach's Concept of the Religious Act}

\section{Summary}

In 1914, the year World War I broke out, Adolf Reinach, one of Edmund Husserl's most notable students, a teacher and mentor of the young generation of phenomenologists, abandoned his work at the university and enlisted in the army. At the front, as a result of various experiences, he went through a vehement turn to religion. He left traces of his conversion in the notes about the religious act he made most probably while in the trenches. In them, Reinach conducted a thorough

${ }^{52}$ E. Husserl, Adolf Reinach $\uparrow$. Nachruf, in: Frankfurter Zeitung 6.12.1917, hier in: Husserliana XXV, Aufsätze und Vorträge (1911-1921), herg. T. Nenon/Seep, H Rainer, Dordrecht 1987, 303.

${ }^{53}$ A. Reinach, Sämtliche Werke: Textkritische Aufgabe in 2 Bänden (Aufzeichnungen), op.cit., S. 595.

${ }^{54}$ H. Conrad-Martius, Einleitung, op.cit., S. XXVII. 
analysis of the human encounter with the Absolute, discussing the conditions for the opening up of the finite being to infinity and the detailed structure of the religious act. The latter, according to the phenomenologist, is constituted in the feeling consisting in the sense of dependence and safety experienced in the presence of God and the response to it, the sense of gratitude and trust. The conclusion of the whole act is the moment of affirmation of the human being who "in" God finds peace and happiness, not desiring to aspire to or strive for anything else.

\section{Keywords}

phenomenology, religious act, mystery, feeling

\section{Slowa kluczowe}

fenomenologia, akt religijny, zagadka, uczucie 